\title{
Universal Properties of Many-Body Delocalization Transitions
}

\author{
Andrew C. Potter, ${ }^{1}$ Romain Vasseur, ${ }^{1,2}$ and S. A. Parameswaran ${ }^{3,4}$ \\ ${ }^{1}$ Department of Physics, University of California, Berkeley, California 94720, USA \\ ${ }^{2}$ Materials Science Division, Lawrence Berkeley National Laboratories, Berkeley, California 94720, USA \\ ${ }^{3}$ Department of Physics and Astronomy, University of California, Irvine, California 92697, USA \\ ${ }^{4}$ California Institute for Quantum Emulation (CAIQuE), Elings Hall, University of California, \\ Santa Barbara, California 93106, USA
}

(Received 15 June 2015; published 14 September 2015)

\begin{abstract}
We study the dynamical melting of "hot" one-dimensional many-body localized systems. As disorder is weakened below a critical value, these nonthermal quantum glasses melt via a continuous dynamical phase transition into classical thermal liquids. By accounting for collective resonant tunneling processes, we derive and numerically solve an effective model for such quantum-to-classical transitions and compute their universal critical properties. Notably, the classical thermal liquid exhibits a broad regime of anomalously slow subdiffusive equilibration dynamics and energy transport. The subdiffusive regime is characterized by a continuously evolving dynamical critical exponent that diverges with a universal power at the transition. Our approach elucidates the universal long-distance, low-energy scaling structure of many-body delocalization transitions in one dimension, in a way that is transparently connected to the underlying microscopic physics. We discuss experimentally testable signatures of the predicted scaling properties.
\end{abstract}

DOI: 10.1103/PhysRevX.5.031033

\section{INTRODUCTION}

The laws of thermodynamics can break down in disordered quantum systems that are isolated from external heat sources because of the localization of excitations that would ordinarily transport energy among distant regions to establish thermal equilibrium [1-4]. Such many-body localized (MBL) systems have the remarkable property that almost all high-energy excited states behave like zero-temperature quantum ground states. Their spectrum can be labeled by an extensive set of local conserved quantities [5,6], such that eigenstates exhibit boundary-law scaling [7] of entanglement entropy, characteristic of gapped quantum ground states. This raises the intriguing possibility that quantum coherent phenomena, typically associated with zero-temperature systems, can occur in arbitrarily "hot" matter. Examples of such quantum coherent phenomena include symmetry breaking below the equilibrium lower critical dimension [8], topological edge states [8-12], and quantum criticality [13-15]. MBL systems also host novel out-of-equilibrium dynamical phase transitions, where thermodynamics breaks down sharply at a critical point [16-19]. Cold atomic, molecular, and trapped-ion systems offer a promising experimental platform to explore these theoretical ideas. Indeed,

Published by the American Physical Society under the terms of the Creative Commons Attribution 3.0 License. Further distribution of this work must maintain attribution to the author(s) and the published article's title, journal citation, and DOI.
Subject Areas: Atomic and Molecular Physics, Condensed Matter Physics, Statistical Physics

issues of thermalization and excited-state dynamics necessarily arise in such systems, as they are inherently well isolated from their surroundings and typically cannot be cooled to low temperatures (compared to their characteristic energy scales).

Theoretical investigations into these questions, however, must tackle a daunting combination of out-of-equilibrium quantum dynamics, interactions, and disorder. Consequently, most existing theoretical work on MBL systems has been confined to small-scale numerics and analysis of phenomenological models. Remarkably, because of the short localization length and short-range entanglement structure of MBL eigenstates deep within the localized phase, these approaches have met with considerable success in gleaning properties of the MBL phase itself. In contrast, these same techniques are poorly suited to access the universal properties of disordered criticality and dynamical phase transitions out of the localized phase.

For example, whereas the ground states of disordered one-dimensional (1D) systems are always localized, the excited states are only many-body localized for sufficiently strong disorder, and they melt into a self-thermalizing incoherent classical fluid at weaker disorder. These two dynamical phases are separated by an apparently continuous many-body delocalization phase transition [4]. Such excited-state delocalization transitions are neither classical thermal phase transitions nor zero-temperature quantum phase transitions but rather represent a novel class of dynamical quantum-to-classical criticality. Such transitions 
are driven by long-distance properties whose characteristic length scale diverges as the critical point is approached, rendering traditional numerical methods increasingly unreliable near the critical point. For example, recent numerical studies [18] of a many-body delocalization transition obtain critical exponents that contradict fundamental bounds [20], suggesting that small-scale numerics cannot access the true long-distance scaling properties. New approaches are clearly needed to understand the nature of this new class of dynamical transitions.

To this end, we develop an effective model for the formation of collective many-body resonances that destabilize the MBL phase at weak disorder, in order to compute the universal scaling properties of many-body delocalization transitions. While the problem of identifying generic many-body resonances is as hard as directly solving the full quantum problem, at any continuous phase transition, one expects a self-similar hierarchical scaling structure. This suggests that the critical resonant cluster may be constructed hierarchically in the spirit of the renormalization group (RG). We implement an efficient numerical procedure to identify the formation of such delocalizing resonant clusters and study the critical properties of the resulting effective model. Our construction is motivated in part by the requirement that the MBL transition itself must be thermal [21] and, thus, incoherent and classical. Accordingly, the critical properties should be well described by an effective classical statistical mechanics model, which, e.g., ignores quantum interference effects such as those responsible for weak localization of noninteracting fermions in equilibrium settings.

We obtain scaling results for the critical properties of the disorder-strength-tuned MBL transition in one dimension, by numerical simulation of the resonance model. We find a continuous dynamical phase transition characterized by a diverging length scale $\xi$, in which a quantum MBL glass melts into a classical thermal liquid. The predicted phase diagram and crossover structure of this effective model are summarized in Fig. 1.

The effective resonance percolation model also provides insight into the dynamics of thermal transport and entanglement of the delocalized thermal liquid, near the melting transition (see Fig. 1). In one dimension, we find that transport on the delocalized side of the transition is anomalously slow, characterized by power-law subdiffusion with a continuously evolving dynamical exponent $z$, which diverges in a universal fashion at the MBL transition. We argue that this subdiffusion stems from tunneling through rare insulating regions, in agreement with recent numerical studies $[16,17]$ and a phenomenological renormalization-group approach [19], and we establish a scaling relation between the correlation length exponent $\nu$, characterizing the divergence of the many-body localization length $\xi$, and the exponent governing the divergence of $z$. We explain how these experimental predictions can be

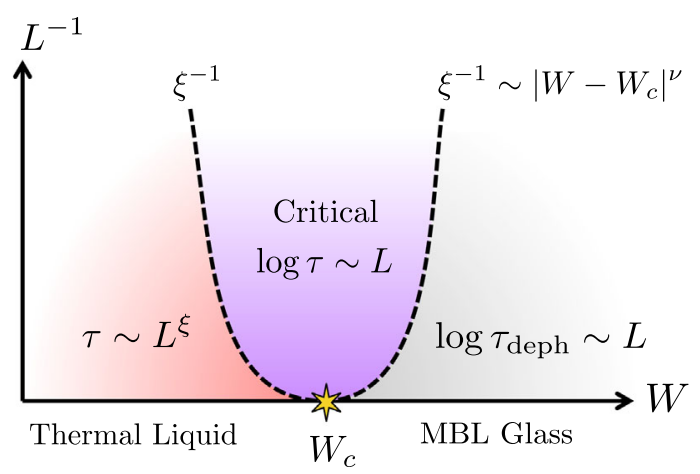

FIG. 1. Phase diagram and finite-size crossovers for onedimensional many-body delocalization transitions. $W$ parametrizes disorder strength. For strong disorder $W>W_{c}$, a nonergodic many-body localized glass is obtained, which lacks thermal transport but exhibits slow dephasing and entanglement dynamics with length-time scaling $L \sim \log \tau_{\text {deph }}$ (where the subscript "deph" emphasizes that this is the dephasing time associated with virtual quantum fluctuations rather than the energy transport time scale, which is strictly infinite in the strong-disorder glass). Below a critical disorder strength $W_{c}$, the MBL glass melts into a thermal, energy-conducting liquid. The melting transition is continuous (second order), characterized by a single diverging length scale $\xi \sim\left|W-W_{c}\right|^{-\nu}$, where $\nu \approx 3.5 \pm 0.3$. Energy transport (entanglement spreading) in the thermal liquid is characterized by a subdiffusive (sub-ballistic) power-law scaling between time and length $\tau \sim L^{z}$, with dynamical exponent $z \sim$ $\xi$ that diverges continuously upon approaching the transition.

tested in cold-atom experiments such as those recently reported in Ref. [22].

This 1D MBL transition was recently studied by meanfield methods [23], exact diagonalization [16-18,24], and a phenomenological renormalization-group procedure [19]. Exact diagonalization studies on 10-20 site chains observe an apparently continuous transition, but they obtain a correlation-length exponent that is incompatible with fundamental bounds [20], suggesting that the observed scaling properties are likely finite-size artifacts that do not reflect the true long-distance universal properties of the MBL transition. In contrast, the critical exponents obtained in our approach comply with the Harris-Chayes bounds [20].

Our results are broadly consistent with those of Ref. [19], where available. We also comment on possible extensions of our results to many-body delocalization transitions in higher dimensions or in systems with long-range interactions-including critical analogs of MBL phases that arise, for example, in random-bond spin chains [13-15,25].

\section{MODEL AND RESONANCES}

Though our effective model for the MBL transition is largely insensitive to the microscopic choice of a particular model, for concreteness, we phrase our discussion in terms of a paradigmatic MBL model, the random-field $X X Z$ chain: 
$H_{\mathrm{MBL}}=\sum_{i}\left[-J\left(\sigma_{i+1}^{x} \sigma_{i}^{x}+\sigma_{i+1}^{y} \sigma_{i}^{y}\right)-\mu_{i} \sigma_{i}^{z}+V \sigma_{i}^{z} \sigma_{i+1}^{z}\right]$.

Here, $\sigma^{x, y, z}$ are Pauli matrices for spin- $1 / 2$ degrees of freedom. This $X X Z$ chain maps, via a standard JordanWigner transformation, into a chain of spinless interacting fermions, where $J$ is the hopping amplitude, $V \ll J$ is the interaction strength, and $\mu_{i}$ is the chemical potential uniformly distributed on the interval $[0, W]$, where $W$ serves as a measure of disorder, and we use the spin and fermionic languages interchangeably. We work in the Fock basis of single-particle orbitals, $\phi_{i}$, of the noninteracting part $(V=0)$ of (1), which have energy $\varepsilon_{i} \approx \mu_{i}+\mathcal{O}\left(J^{2} / W\right)$ and are localized near site $i$. We then consider the interactions $V$ as a perturbation expressed in this basis, though we remark that our method is not confined to low-order perturbation theory but, rather, includes contributions from all orders in $V$. We are interested in the properties of typical highly excited states in the middle of the many-body spectrum. This Hamiltonian has been considered as a prototypical example of a MBL system (see, e.g., Ref. [26] for a recent review), characterized by the absence of thermal transport and by a slow, logarithmic spreading of entanglement due to dephasing $[6,27,28]$. When $V \neq 0$, the system is believed to exhibit a MBL-to-thermal dynamical phase transition as a function of disorder strength [16-19], which separates a nonergodic MBL quantum glass phase from a ergodic high-temperature classical liquid.

To motivate the problem of delocalization, imagine creating an energy wave packet initially localized near a single site in the MBL system. How does such a packet evolve with time? Deep in the localized phase, it is extremely unlikely that it can tunnel from a given site (bond) to another because of the wide variance of site (bond) energies. More precisely, the amplitude to tunnel through a distance $x$ of the localized phase is

$$
\Gamma(x) \approx V e^{-x / x_{0}},
$$

where $x_{0} \approx\left[\frac{1}{2} \ln \left(1+(W / J)^{2}\right)\right]^{-1}$ is the single-particle localization length (measured in units of the lattice spacing), where we have chosen an expression that interpolates smoothly between the strong-disorder limit $x_{0} \approx \ln ^{-1}(W / J)$ and the weak-disorder limit $x_{0} \approx(J / W)^{2}$. If the tunneling strength $\Gamma\left(x_{i j}\right)$ between spin pairs $i$ and $j$ separated by a distance $x_{i j}$ is much smaller than their energy difference $\delta E_{i j}$, then the true many-body eigenstate is very close to a product state of independent configurations of each bond. On the other hand, if $\Gamma\left(x_{i j}\right) \gtrsim \delta E_{i j}$, then the many-body eigenstate contains an entangled superposition of the degrees of freedom on the two sites, and we say that the two sites are resonantly linked. We estimate the probability that a given site is resonantly linked to at least one other as $\left(1-e^{-\lambda_{R}}\right)$, with
$\lambda_{R}=\nu(0) \int_{0}^{\infty} d x \Gamma(x) \approx(V / J) x_{0}$, where $\nu(0) \approx 1 / J$ is the single-particle density of states.

For very strong disorder $\left(x_{0} \ll 1\right)$, the density of resonantly linked pairs is very small, $\rho_{\text {res }} \approx \lambda_{R} \ll 1$, so that the resonant links are well isolated from each other, and they do not disrupt the properties of the surrounding localized phase. As disorder is weakened, these resonant links occur more frequently and eventually disrupt localization below a critical disorder strength $W_{c}$. Since, in a thermal phase, all spins are highly interentangled, delocalization and the recovery of thermalization at weak disorder must occur via a highly collective resonance involving every single spin. Identifying all possible generic manybody resonances is a difficult problem, and in fact, it is as difficult as exactly solving the full quantum many-body problem. However, near the delocalization critical point, it is natural to expect that the critical resonant cluster exhibits scaling structure that is geometrically self-similar (in a statistical sense) at different energy scales. Anticipating this hierarchical structure motivates a simpler way of identifying collective resonances by first identifying small resonant clusters (e.g., resonant pairs as above) and then examining whether groups of these small resonant clusters can collectively resonate on some lower energy scale (longer time scale), and so on. Using this perspective, we construct an effective quantum percolation model for delocalization by hierarchical collective resonances.

\section{EFFECTIVE MODEL FOR THE DELOCALIZATION TRANSITION}

A cluster of $m$ resonantly linked sites is characterized by bands of $\mathcal{N} \approx 2^{m}$ many-body energy levels and thus has an exponentially small level spacing in $m$. Roughly speaking, collective rearrangements among spins of two such clusters containing $m_{1,2}$ sites, respectively, and separated by distance $L_{12}$ can occur resonantly if the effective tunneling $\Gamma_{12}$ is larger than the level spacing of the combined cluster, scaling as $\sim 2^{-\left(m_{1}+m_{2}\right)}$. We therefore see that this exponential suppression of the level spacing will favor large, manyspin resonant clusters. This motivates an effective model for the delocalization transition, consisting of the following iterative procedure:

(1) Examine a disordered chain to identify resonantly linked pairs of spins and group them into two-spin clusters.

(2) Identify the strongest resonantly coupled pair of clusters (i.e., the one with the largest interaction $\Gamma_{i j}$ ) and merge them into a new, larger resonant cluster.

(3) Account for the reduction of level spacing on the newly formed resonant cluster.

(4) Compute the effective interaction of the new cluster with other clusters (as described in detail below).

(5) Examine whether the reduced level spacing enables any of the newly formed clusters to resonantly interact; if so, merge these clusters. 
Steps 2-5 can then be iterated (numerically) until all possible clusters are formed.

Before giving a detailed account of this procedure, we briefly sketch its potential outcomes and underlying philosophy. For strong disorder, the resonance merging ceases after a small number of steps, producing only dilute, wellisolated, small resonant clusters embedded inside strongly localized quantum glass. Upon weakening disorder, the size of the resonant clusters increases, diverging continuously at a critical value of disorder $W=W_{c}$, where a single cluster just barely percolates across the system (in the limit of infinite system size). For weaker disorder $\left(W<W_{c}\right)$, the resonance-merging procedure continues until all degrees of freedom are subsumed into a single percolating cluster (in the infinite system limit), resulting in a delocalized thermal phase. Nevertheless, we see that the near-critical transport properties of the delocalized phase exhibit anomalously slow dynamics, with an unusual scaling structure, because of the proximity to the quantum glass phase.

While we have described the resonance-merging procedure as a static process to identify resonant structures in the Hilbert space of the disordered problem, we can also give a dynamical interpretation of the procedure (analogous to the relation between equivalent Hilbert-space [14,15] and dynamical [13] real-space RG approaches to describe excited states of random critical systems). In this picture, resonances arise from a small mismatch in energy scales, corresponding to a slow beating frequency or long time in dynamical processes. Over the coarse of iterations, the dynamical time scales associated with the merging of resonances steadily lengthen. In this way, the effective model closely resembles a renormalization-group procedure - first accounting for fast degrees of freedom and progressively proceeding to slower and slower modes.

We also remark that, in principle, the problem of identifying generic many-spin resonances is factorially complicated in system size and hence is no simpler than directly solving the full quantum problem. From our analytic treatment of the strong-disorder phase, we expect that the most likely type of resonances are those between two bonds (orbitals) motivating our use of step 1) above to "seed" the iterative resonancemerging procedure. Moreover, near the transition, whether or not a resonant cluster percolates across the system is driven by highly collective resonances among large clusters of many spins, and hence the low-energy, long time-scale dynamics of the near-critical cluster are expected to be insensitive to the details of early steps in the procedure (i.e., are universal).

\section{A. Criterion for merging resonant clusters}

To test if two clusters $i$ and $j$ can resonate (step 2), we compare their coupling $\Gamma_{i, j}$ to the energy mismatch $\delta E_{i, j}$ of their energy levels. For two large clusters $i, j$, such that the bandwidth of each cluster exceeds the level spacing of the other $\left(\min \left\{\Lambda_{i}, \Lambda_{j}\right\}>\max \left\{\delta_{i}, \delta_{j}\right\}\right)$, we define an energy mismatch $\delta E_{i, j}$ between two clusters $i, j$ as follows: $\left(\delta_{i} \delta_{j} / \min \left\{\Lambda_{i}, \Lambda_{j}\right\}\right)$. In the other case, where one cluster's bandwidth is smaller than the other's level spacing, we define $\delta E_{i, j}=\max \left\{\delta_{i}, \delta_{j}\right\}-\min \left\{\Lambda_{i}, \Lambda_{j}\right\}$ (see Appendix A 1 ). We take the bandwidth of the newly merged cluster to be $\Lambda_{i \cup j}=\Lambda_{i}+\Lambda_{j}+\Gamma_{i, j}$, and the level spacing to be $\delta_{i \cup j}=\Lambda_{i \cup j} /\left(2^{m_{i}+k_{j}}-1\right)$. We emphasize that the main ingredient at this step is the exponential reduction of the level spacing when two clusters are merged: We checked numerically that the universal properties of the transition are insensitive to the other details of the procedure.

\section{B. Renormalization of intercluster couplings}

After merging two clusters, one should, in principle, compute a new set of intercluster couplings (step 4 above). Consider merging two clusters $i$ and $j$, with number of spins $m_{i}, m_{j}$ and bandwidths $\Lambda_{i}, \Lambda_{j}$, respectively, interacting with coupling $\Gamma_{i, j}$. In order for a third cluster, $k$, to drive a collective rearrangement of all $2^{m_{i}+m_{j}}$ levels of the merged cluster, $i \cup j$, and hence access the full many-body level spacing of $i \cup j, k$ must interact separately with both $i$ and $j$. The effective interaction strength for such a process depends on the energy mismatch between $k$ and $i, j$, respectively, and can occur by several different possible processes. For instance, consider the process $k \rightarrow i \rightarrow j$, where $k$ first interacts with $i$ and then $i$ with $j$. If the energy mismatch $\delta E_{k, i / j}$ between $k$ and $i$ or $j$ is large compared to the couplings $\Gamma_{k, i / j}$ between $k$ and $i$ or $j$, then the interaction between $k$ and $i \cup j$ can be computed perturbatively:

$$
\Gamma_{k, i \cup j}^{(k \rightarrow i \rightarrow j)} \approx \frac{\Gamma_{k, i} \Gamma_{i, j}}{\delta E_{k, i}} .
$$

In the alternative case where all three clusters $i, j$, and $k$ can resonate, e.g., $\Gamma_{k, i}>\delta E_{k, i}$, we may estimate the effective coupling between $k$ and $i \cup j$ by classically adding the time taken for an excitation to transfer from $k \rightarrow i \rightarrow j$ :

$$
\Gamma_{k, i \cup j}^{(k \rightarrow i \rightarrow j)} \approx\left(\Gamma_{k, i}^{-1}+\Gamma_{i, j}^{-1}\right)^{-1} .
$$

There are a few possible routes for $k$ to excite both $i$ and $j-$ for example, $k$ can first excite $i$, and then $i$ excites $j$, or $k$ can excite both $i$ and $j$, etc. We compute $\Gamma_{k, i \cup j}$ for each process using the "perturbative" or "classical" rules above as appropriate, and choose their maximum as the new effective coupling between $k$ and $i \cup j$.

Computing the renormalized intercluster couplings in step 4 is conceptually important to avoid a potential instability of the strong-disorder phase (see Appendix A); however, in practice, we find that this instability is absent for all numerically accessible system sizes, and we find identical scaling independent of whether or not the intercluster couplings are renormalized. We remark that the way the couplings are renormalized is reminiscent of the approach of Ref. [19], where metallic and isolating blocks are merged by using a renormalization group scheme. However, we point out that 
we only merge "metallic" (resonant) clusters in our model, thus avoiding the thorny issue of determining the proper renormalized couplings after merging "mixed" insulating and thermalizing blocks.

\section{Validity of quantum percolation model}

Before proceeding, we pause to comment on the assumptions and approximations that have gone into constructing the effective quantum percolation model of the delocalization transition. Our key assumption is that the critical resonant cluster has a self-similar structure in energy and space, which allows us to hierarchically construct the percolating resonance that drives delocalization. This hierarchical construction clearly does not describe the most general collective resonances and, in particular, ignores generic unstructured resonant clusters that do not decompose into a hierarchical tree of few-body resonances. However, the assumption of self-similarity is naturally expected for a continuous phase transition characterized by a diverging length scale, based on knowledge of more conventional disordered criticality. We therefore expect our approach to accurately capture the universal aspects of such a resonant structure close to the delocalization transition.

Our approach also seeks to construct a delocalizing resonance by merging metallic clusters. Such an approach is known to be problematic for noninteracting Anderson insulators in which the conductivity of blocks does not add in a simple fashion because of coherent interference effects [29]. However, in the present context, since the system must be thermal at the MBL transition, we expect strong dephasing to wash out any effects of quantum interference (e.g., the time scale for dephasing is more rapid than that of transport and does not diverge even in the MBL phase).

Lastly, we remark that while we are implicitly working at infinite effective temperature (e.g., we assume, for simplicity, that all $2^{n}=e^{n \log 2}$ many-body levels associated with an $n$-spin cluster are equally accessible, equivalent to having maximal entropy per spin of $\log 2$ ), we stress that this assumption is purely a matter of convenience, and we expect the same universal structure at any nonzero energy density. Specifically, we have numerically checked that changing the amount of thermal entropy per cluster does not affect the critical exponents of the transition.

\section{SCALING STRUCTURE OF THE MBL TRANSITION}

To identify the scaling structure of the transition predicted by the effective resonance percolation model described above, we examine the dependence of the size, $\xi_{\text {loc }}$ (number of spins), of the largest resonant cluster as a function of disorder strength $W$ for various system sizes $L$. Disorder-averaged results for $V=0.3$ are shown in Fig. 2. For $W \ll W_{c}$, one obtains a percolating cluster with $\xi_{\text {loc }} \approx L$ with probability close to 1 . In the finite-size

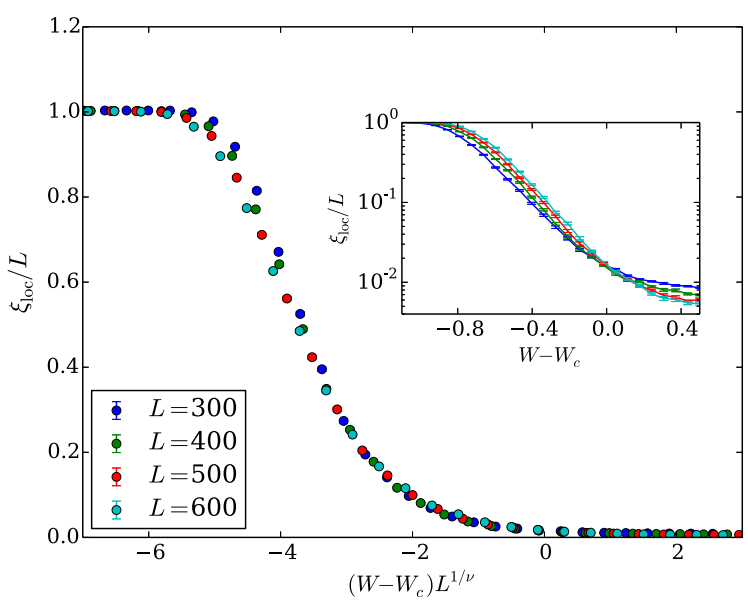

FIG. 2. Finite-size scaling for the disorder-averaged localization length $\xi_{\text {loc }}$ (defined as the size of the longest resonant cluster for a given disorder realization). $\xi_{\text {loc }} / L$ collapse to a universal form (main panel) when plotted against the scaling variable $\left(W-W_{c}\right) L^{1 / \nu}$, with correlation length exponent $\nu \approx 3.5 \pm 0.3$. Insets show the unscaled $\xi_{\text {loc }} / L$ curves. Results are averaged over $\sim 10^{4}$ disorder realizations.

systems simulated, $\xi_{\text {loc }} / L$ crosses over from $1\left(W \ll W_{c}\right)$ to zero $\left(W \gg W_{c}\right)$ as disorder is increased.

The curves for different system sizes cross at the same value of disorder strength, which we identify as the critical disorder strength $W_{c}$. Moreover, the data for different system sizes collapse to a universal scaling form $\xi_{\text {loc }}=L \Xi\left(\left(W-W_{c}\right) L^{1 / \nu}\right)$, with correlation length exponent $\nu=3.5 \pm 0.3$, as shown by plotting $\xi_{\text {loc }} / L$ against the scaling variable $\left(W-W_{c}\right) L^{1 / \nu}$ (Fig. 2). Here, $\Xi(x)$ is a universal scaling function interpolating between $1(x \ll 0)$ and zero $(x \gg 0)$.

This scaling collapse indicates the existence of a length scale $\xi$ that diverges when the transition is approached from either side as $\xi \sim\left|W-W_{c}\right|^{-\nu}$, signaling a sharp continuous delocalization phase transition. The diverging length scale $\xi$ can be interpreted as the localization length for $W>W_{c}$ and, as we will see below, characterizes the length of insulating gaps in the transport path for $W<W_{c}$.

We remark that the (universal) value of the scaling function at criticality, $\Xi(0) \approx 10^{-2}$, is anomalously low compared to ordinary percolation [for which $\Xi(0)$ would be $\approx 0.5]$. This strong asymmetry indicates that the transition is driven by rare resonant clusters that are quite sparse and widely separated (e.g., clusters of size $\ell$ are separated by typical distance $\approx \Xi(0)^{-1} \times \ell \gg \ell$ at criticality).

We also performed simulations of a simpler model that ignores the renormalization of the couplings when merging clusters. As claimed above, despite the potential stability issues of the strong-disorder phase within that simplified model, we find essentially identical universal percolation curves using these simplified rules, compatible with the 
same universal exponent $\nu \approx 3.5$ within error bars (see Appendix A).

\section{NEAR-CRITICAL DYNAMICS IN THE DELOCALIZED PHASE}

We now turn to the task of computing the scaling of transport and entanglement dynamics, in the delocalized phase near the critical point $\left(W \lesssim W_{c}\right)$. Our goal is to understand the scaling structure underpinning the critical slowing down of energy transport as $W$ approaches $W_{c}$ from below. Since the delocalized phase is thermal and at high temperature [21], transport occurs via the thermally incoherent transfer of energy among bonds in the resonant cluster. Hence, we model the spread of excess energy (initially localized near position $x$ as a function of time) as a classical random walk across the resonant cluster, with a time scale $\tau_{A B}=1 / \Gamma_{A B}$ to transfer excitations between resonantly coupled clusters $A$ and $B$. Namely, we can write a stochastic transition matrix $M_{i j}=\Gamma_{i j}-\delta_{i j} \sum_{k} \Gamma_{i k}$ and compute the time evolution of the mean-square displacement of a wave packet (averaged over initial position) as $\delta r^{2}(t)=(1 / L) \sum_{i j}\left(e^{M t}\right)_{i j}\left(x_{i}-x_{j}\right)^{2}$.

The iterative merging procedure yields detailed information about the connectivity structure and the coupling strengths for each participating link in the resonant cluster. Here, it is important to note that, while all degrees of freedom are part of the percolating resonant cluster for $W<W_{c}$, not all spins contribute equally to transport and dynamics. Rather, dynamical properties are dominated by a subset of efficiently connected spins that form the "backbone" of the transport path through the resonant cluster. Then, using standard Green's function methods, we compute the time evolution of an initially well-localized energy wave packet spreading across the resonances network via a random walk. Our main result is that the delocalized phase exhibits a broad regime of anomalously slow subdiffusive equilibration dynamics and energy transport. We observe this subdiffusive behavior in our model using both renormalized and nonrenormalized couplings, but find that finite-size effects are much weaker for the simplified model that ignores renormalization, thus allowing us to extract much cleaner data. Specifically, the renormalization produces a numerically large separation of scales for short-distance (weakly renormalized) and long-distance (heavily renormalized) intercluster couplings that exacerbate numerical precision limits for exponentiating the stochastic time evolution matrix $e^{M t}$ at large times. Since these two results give apparently identical scaling results on accessible system sizes (recall that they reproduce equally well the universal scaling curves for $\xi_{\text {loc }} / L$ described in the previous section), we instead focus on this simplified model [30].

Averaging over initial position $x$, and over disorder configurations, we find that the mean-square displacement of an excitation grows as a power law in time (Fig. 3),

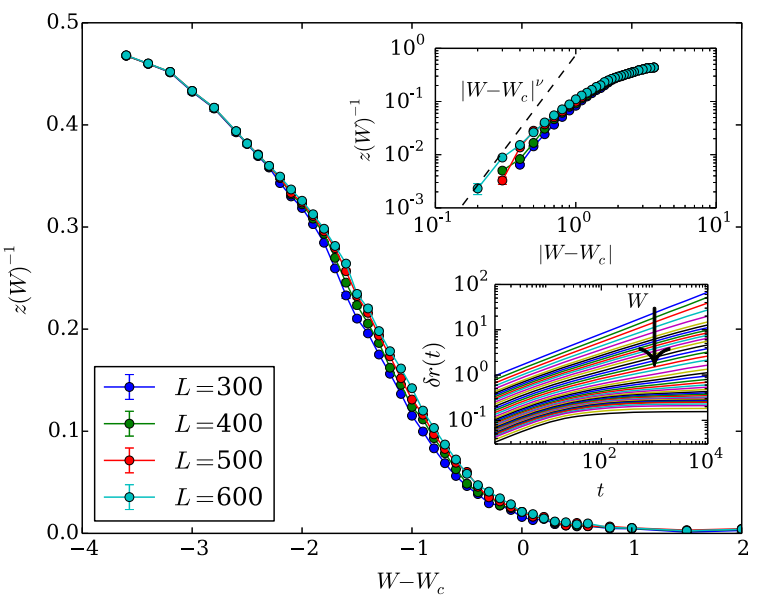

FIG. 3. Energy transport near the transition. In the delocalized phase $\left(W<W_{c}\right.$ ), excess energy initially localized near the origin spreads to a distance $\delta r \sim t^{1 / z}$ in time $t$ (bottom inset). The dynamical critical exponent $z$ diverges continuously from the delocalized side of the transition and vanishes inside the quantum critical glass phase (main panel). The numerical results are consistent with $z$ diverging as $\left|W-W_{c}\right|^{-\zeta}$ with $\zeta=\nu$ (top inset).

$$
\overline{|\delta r(t)|} \approx t^{1 / z(W)}
$$

For ordinary classical diffusion, $z=2$. In contrast, we find that $z(W)$ diverges in the glassy phase, indicating an absence of energy transport and a breakdown of thermal equilibration. On the delocalized side of the critical point, $1 / z(W)$ increases continuously from zero as a function of detuning from the critical disorder strength:

$$
z(W) \approx \frac{z_{0}}{\left(W_{c}-W\right)^{\zeta}} \quad\left(W \lesssim W_{c}\right),
$$

where $z_{0}$ is a nonuniversal constant. Though seemingly a distinct universal exponent, $\zeta$ is related by a general scaling relation to the log-dynamical exponent $\psi$ of the localized phase and the correlation length exponent $\nu$. To see this, note that at the critical point, transport along the critical resonant chain occurs by tunneling through arbitrarily long localized regions; hence, energy scales with length as $\sim e^{-L}$ at the critical point, just as in the localized phase. This scaling must cross over smoothly to the power-law scaling of Eq. (5) on length scales $L \approx \xi$, implying the scaling relation

$$
\zeta=\nu
$$

Using this scaling relation and the definition of the correlation-length exponent, we may rewrite Eq. (6) as $z \sim$ $\xi$ (ignoring logarithmic corrections and nonuniversal prefactors). Accessing the scaling regime (6) to measure $\zeta$ numerically is difficult because of the form of the finite-size percolation curves (Fig. 2): Close to the critical point with $W<W_{c}, \xi_{\text {loc }}>L$, even for moderately large systems. 
In particular, in our finite-size simulations, about $1 \%$ of samples have a percolating transport path even on the localized side of the transition, $W \gtrsim W_{c}$, consistent with the observed $z^{-1} \sim 10^{-2}$. In contrast, no configurations will percolate for $W>W_{c}$ in an infinite system. Since longdistance transport requires a percolating transport path, $z^{-1}$ will vanish for $W>W_{c}$, indicating that the small residual $z^{-1}\left(W \geq W_{c}\right)$ observed is a finite-size artifact. Upon subtracting the value of $z\left(W_{c}\right)^{-1}$, we find critical behavior compatible with the expected scaling relation $\zeta=\nu$ (Fig. 3, upper inset). We caution that, since the transport is sensitive to whether or not the cluster percolates, and given the small percolation probability at criticality, $\xi_{\text {loc }} / L \sim$ $10^{-2}$ (a universal number insensitive to model details), transport generally suffers more severe finite-size corrections than, say, $\xi_{\text {loc }}$.

\section{A. Structure of transport path}

Subdiffusive transport arises from the broad distribution of effective tunneling links, $\Gamma_{i j}$, in the resonant backbone, corresponding to a power-law distribution of time scales $[17,31], \tau_{i j} \sim 1 / \Gamma_{i j}$ :

$$
p(\tau) \sim \frac{1}{\tau^{\alpha}},
$$

with $1<\alpha \leq 2$ such that the mean waiting time $\int{ }^{\infty} \tau p(\tau) d \tau$ is divergent. To see the relation between this power-law distribution and subdiffusive transport properties, note that energy transport occurs as a random walk on a onedimensional chain whose "sites" are two-spin bonds in the resonant cluster and whose links are weighted by a waiting time $\tau$ corresponding to the inverse effective coupling between "sites." (In principle, the bonds also have variable lengths, $\ell \sim \log \tau$. However, unlike the waiting times, the bond lengths have a finite mean $\bar{\ell}$, and in the following, we approximate the length of each step as $\bar{\ell}$ ). In $N$ steps, a random walker traverses $\sim \mathcal{O}(\sqrt{N})$ different bonds, revisiting each approximately $\sqrt{N}$ times and moving a total distance $L \approx \sqrt{N}$ (in units of the average step size). For a broad distribution of waiting times, the time taken for $N$ steps is dominated by the longest waiting time $T$ encountered. The probability of encountering a bond with a waiting time of at least $T$ is $P(\tau \geq T)=\int_{T}^{\infty} p(\tau) \approx T^{1-\alpha}$. We can reasonably expect to find a link with waiting time $\tau \gtrsim T$ among $L \approx \sqrt{N}$ different bonds if $L P(\tau \geq T) \approx 1$, and therefore, the slowest link in a region of size $L$ has waiting time $T(L) \approx L^{1 /(\alpha-1)}$. Since this weak link is revisited order $\sqrt{N} \approx L$ times, the total time to move distance $L$ scales as $t_{\text {energy }}(L) \approx L^{\alpha /(\alpha-1)}$. Comparing to Eq. (5), we identify the dynamical exponent for energy transport as

$$
z \equiv z_{\text {energy }}=\frac{\alpha}{\alpha-1},
$$

implying that $\alpha$ approaches 1 near the transition as $\alpha(W)-1 \sim\left|W-W_{c}\right|^{\zeta}$.

Note also that the broad distribution of waiting times (8) corresponds to a probability of encountering a link of length $\ell=\log \tau$ in the transport path along the resonant backbone, near the transition, given by

$$
P(\ell) \sim e^{(1-\alpha) \ell} \sim e^{-l / z}=e^{-(\ell / \xi)} .
$$

Hence, the typical spacing between "vertebrae" in the resonant backbone is $\approx \xi$, corresponding to an energy scale $e^{-\xi}$. However, in a region of length $L \gg \xi$, it is extremely unlikely to avoid encountering an atypically long gap of length $\ell_{\star}$, defined by $(L / \xi) P\left(\ell_{\star}\right) \sim 1$, i.e., $\ell_{\star} \sim \xi[\log (L / \xi)]$. Such long rare links involve a waiting time $\tau \sim e^{l_{\star}} \sim L^{\xi}$, which dominates the time required to traverse a segment of size $L$.

\section{B. Entanglement dynamics vs thermal transport}

We note that the dynamics of energy transport scale differently than those of entanglement since energy is a conserved quantity that can only be transferred among subregions, whereas entanglement can be freely generated $[19,32]$. Hence, whereas energy transport can be viewed as a random walk of conserved particlelike excitations, entropy spreads deterministically in all directions simultaneously [32]. In order to entangle regions separated by distance $L$, entanglement must spread across order $L$ links, only visiting each once (in the same spirit as the second law of thermodynamics, once a bond is entangled with many others, it is extremely unlikely to later disentangle itself). Therefore, the time scale for entanglement spread is dominated by the longest typical waiting time encountered, $t_{\mathrm{ent}} \approx T(L) \approx$ $L^{1 /(\alpha-1)}$. In comparison, we find a different effective dynamical exponent for the spread of entanglement:

$$
z_{\mathrm{ent}}=\frac{1}{\alpha-1}=z_{\mathrm{energy}}-1,
$$

with $z_{\text {ent }} \sim z \sim \xi$ near the transition.

\section{Scaling of optical conductivity}

So far, we have discussed transport from the perspective of the propagation of an initially well-localized energy wave packet. While this is natural in ultracold atomic systems (see, e.g., Ref. [33]), in solid-state contexts one frequently thinks of dynamics in terms of frequencydependent conductivity. The power-law distribution of time scales also implies an anomalous power-law frequency dependence for ac conductivities. For systems with a conserved spin component or particle number, one can compute the scaling of the optical number conductivity via the Einstein relation $\sigma(\omega) \approx D(\omega) \chi$, where $D(\omega)$ is the (frequency-scale-dependent) diffusion constant, and $\chi$ is the static compressibility (which will be constant 
throughout the phase diagram). The diffusion constant scales like $D \sim \omega L^{2} \sim \omega^{1-2 / z}$, where $z=z_{\text {energy }}$ is the dynamical scaling of energy; hence, the optical conductivity will also scale as

$$
\sigma(\omega) \sim \omega^{1-2 / z} .
$$

This expression interpolates between constant conductivity in the diffusive limit $(z=2)$ for weak disorder, to $\sigma\left(W_{c}\right) \sim$ $\omega$ at the MBL transition $z=\infty$. This scaling argument agrees with the recent rare-regions analysis and numerical results of Ref. [17].

\section{SUMMARY AND EXTENSIONS}

\section{A. Phase diagram}

Let us summarize our results in the thermal phase $\left(W<W_{c}\right)$. Near the critical point, the system exhibits anomalously slow subdiffusive dynamics $\tau \sim L^{\xi}$. Inside the critical regime (i.e., on length scales $L \ll\left|W-W_{c}\right|^{-\nu}$ ), the critical resonant cluster mediates coherent transport of energy with characteristic scaling of length and time $\log \tau \sim L$. This scaling relation is reminiscent of the one governing the dynamics of dephasing and entanglement growth in the localized MBL phase, with the important distinction that dynamics in the strong-disorder MBL phase describe only virtual ("off-shell") transitions rather than real ("on-shell," or resonant) processes required for thermal transport. Hence, whereas the MBL glass is nonergodic, the energy eigenstates at the glass-melting critical point are ergodic and thermal, with volume-law scaling of entanglement in every eigenstate (consistent with general entanglement monotonicity requirements [21]). However, while the pure eigenstates at the delocalization transition exhibit thermal behavior, starting from a superposition of energy eigenstates (the only initial conditions that can be prepared experimentally in finite time), the system will take a superpolynomially long time (in system size) to equilibrate and thermalize. The resulting phase diagram and crossover scales are shown in Fig. 1.

\section{B. Experimental signatures}

The scaling structure of the MBL transition predicted by our approach has observable consequences testable with currently existing ultracold atom technology. First, the subdiffusive transport for $W<W_{c}$ is directly observable in wave-packet expansion experiments, such as those used to diagnose Anderson insulators from speckle disorder [34].

In addition, recent experiments [22] observe signs of many-body localization by measuring the decay of an initial density imbalance $\mathcal{I}_{0}$ between even and odd sites [35]. Whereas in a thermalizing system $\mathcal{I}_{0}$ decays to zero at long times, because of the absence of spontaneous symmetry breaking at finite temperatures in $1 \mathrm{D}$, in MBL systems, the density imbalance saturates to a nonzero steady state value $\mathcal{I}_{\infty}$. The saturation value $\mathcal{I}_{\infty}$ is given by the overlap of the initial imbalance with the local conserved quantities of the MBL phase. For $W \gtrsim W_{c}$, the spatial extent of these conserved quantities is $\sim \xi$; i.e., the conserved quantities are highly entangled products of degrees of freedom within a distance $\xi$, and hence their overlap with a generic product state of local operators is $\sim 2^{-\xi / 2}$. Thus, the saturation value decays as

$$
\mathcal{I}_{\infty} \sim \mathcal{I}_{0} e^{-\xi} \sim \mathcal{I}_{0} e^{-\left|W-W_{c}\right|^{-\nu}}
$$

as $W \rightarrow W_{c}$ from above (omitting nonuniversal constant prefactors in the exponentials), enabling an experimental measurement of the correlation-length exponent $\nu$. Moreover, the exponential sensitivity of $\mathcal{I}_{\infty}$ to $\xi$ provides a way to distinguish the interacting many-body localized insulator from a noninteracting localized insulator, for which $\mathcal{I}_{\infty} \sim 1 / \xi^{2}[22]$.

On the thermalizing side of the transition, $\mathcal{I}_{\infty}=0$ as required by ergodicity; however, one may examine the time-dependent decay of $\mathcal{I}(t)$ near the transition. At long times, $t \gg e^{\xi / x_{0}}$ [see Eq. (2)], the initial imbalance $\mathcal{I}_{0}$ is washed out in typical portions of the system. However, since there is a broad distribution of thermalization times, the imbalance persists in rare strongly disordered regions that thermalize much more slowly than average - these are the same regions that serve as bottlenecks for transport, producing subdiffusion. We have seen, from transport simulations, that the probability of finding an insulating region of size $\ell$ and thermalization time $\tau(\ell) \sim e^{\ell / x_{0}}$ is $P(\ell) \sim e^{-\ell / \xi}$ [see Eq. (10)]. Hence, the imbalance after long time $t$ decays as (up to logarithmic corrections and nonuniversal prefactors)

$$
\mathcal{I}(t) \sim \int_{x_{0} \log t}^{\infty} P(\ell) d \ell \sim \frac{1}{t^{1 / z}}
$$

(recall $z \sim \xi$ ). Therefore, we see that the dynamics of population-imbalance decay enables a direct observation of the subdiffusive dynamics of the system. We note that, while we have focused on population imbalance because of its connection with Ref. [22], the above discussion pertains to any generic sum of local observables (e.g., magnetization) that decays to zero in a thermalizing system.

\section{Extension to higher dimensions and long-range interactions}

In addition to the 1D MBL transition studied here, our approach can be adapted to treat a variety of other many-body delocalization transitions, including higherdimensional MBL transitions, MBL transitions with long-range interactions [36], and critical analogs of MBL with stretched-exponential interactions [13-15]. While each of these deserves its own detailed study, our 
analysis of the 1D MBL transition enables us to make several conjectures about their scaling properties.

Similar to the 1D transition, we still expect the higherdimensional MBL transition to be characterized by a single diverging length scale $\xi \sim\left(1 /\left|W-W_{c}\right|^{\nu_{d}}\right)$, with correlationlength exponent $\nu_{d}$ that depends on dimensionality $d$, and $z=\infty$ (tunneling-like) dynamics at criticality associated with a fractal structure to the critical resonant cluster. One clear distinction is that the subdiffusive transport for $W<W_{c}$ is almost certainly special to one dimension. Specifically, in one dimension, subdiffusion arises from rare bottlenecks in the transport path. In contrast, in two and higher dimensions, transport can simply go around such bottlenecks. Hence, in higher dimensions, we expect a nonzero diffusion constant $(z=2)$ and finite dc conductivity in the entire region $W<W_{c}$. Then, repeating the arguments leading to the scaling relation $\zeta=\nu$ above, one would expect the diffusion constant to vanish as $D \sim e^{-\xi / x_{0}}$ upon approaching the higher-dimensional MBL transition.

Regarding long-range interacting or nonergodic critical localized models, one might intuitively expect spatially extended interactions to dramatically modify the scaling of the MBL transitions compared to the short-range interactions studied here. However, simple-minded arguments (see Appendix B) suggest that the long-range two-body interactions are strongly renormalized to produce shortrange many-body interactions between large resonant clusters. This suggests that the scaling properties of the MBL transition may be insensitive to longer-range interactions (so long as the interactions are not sufficiently long range to destabilize the MBL phase [36]). We leave a detailed study of these issues to future work.

\section{DISCUSSION}

To summarize, we have developed an effective model for the many-body delocalization transition of a one-dimensional MBL phase. This model can be efficiently simulated for very large system sizes (with computational cost scaling polynomially in system size), and we argue that it gives an accurate description of its long-distance, low-energy, universal critical properties.

This transition represents a novel form of nonequilibrium excited-state criticality, distinct from classical, thermal phase transitions and zero-temperature quantum criticality. We find that this excited-state transition is continuous (second order), characterized by a single diverging length scale. We describe the set of universal critical exponents that characterize this melting transition, which are connected by scaling relations. In particular, we find an unusual scaling structure of the critical slowing down of dynamics and thermal transport characterized by a continuously evolving dynamical exponent $z$, which diverges in a universal fashion upon approaching the quantum critical point. This unusual, universal divergence of a critical exponent $z$ stems from a sparse structure to the transport path through the critical percolating cluster. It is natural to expect that this sparsity corresponds to a (multi) fractal structure of the critical cluster. While our approach makes many simplifying approximations, and is clearly not exact, we believe the general scaling structure it predicts (Fig. 1) is correct and, moreover, expect to obtain a reasonable (though possibly approximate) value for the correlation-length exponent, $\nu$. In particular, it is encouraging that the scaling data of Fig. 1 are closely compatible with the renormalization-group approach of Refs. [19,37], despite the marked differences between these approaches. Testing these results using standard numerical techniques may be challenging. In particular, a direct frontal assault via exact diagonalization is unlikely to give accurate scaling results in systems of only a few tens of sites.

We anticipate that these methods can be extended to treat a variety of other out-of-equilibrium dynamical phase transitions in disordered quantum systems. For instance, our analysis in terms of quantum percolation of resonant clusters can be straightforwardly adapted to treat higherdimensional delocalization transitions, which are so far inaccessible by other methods.

Another unanswered question regarding one-dimensional systems concerns the fate of subdiffusion deep in the delocalized phase at weak disorder, far from the MBL transition. One possible scenario is that subdiffusion persists to arbitrarily weak disorder, such that diffusion is only asymptotically recovered in the clean limit. This would imply that any amount of disorder in one-dimensional systems leads to subdiffusive dynamics. The alternative would be a second finite-disorder-strength excited-state phase transition separating diffusive and subdiffusive regimes. We leave a detailed investigation of such issues for future work.

\section{ACKNOWLEDGMENTS}

We are grateful to D. Huse and E. Altman for many insightful comments and a detailed comparison of results of Ref. [19]. We also acknowledge helpful conversations with J. E. Moore, A. Vishwanath, R. Vosk, C. R. Laumann, and S. L. Sondhi. This work was supported by the Gordon and Betty Moore Foundation's EPiQS Initiative through Grant No. GBMF4307 (A. C. P.), the Quantum Materials Program at LBNL (R. V.), NSF Grant No. DMR-1455366, UC Irvine startup funds, and the President's Research Catalyst Award No. CA-15-327861 from the University of California Office of the President (S. A. P.).

\section{APPENDIX A: DETAILS OF EFFECTIVE MODEL OF THE DELOCALIZATION TRANSITION}

\section{A. Resonance merging procedure}

In the first stage of resonance formation, we check for pairwise resonances between two spin pairs $i$ and $j$ for which $\Gamma\left(x_{i j}\right)>\delta E_{i j}$, where $\Gamma(x) \approx V e^{-x / x_{0}}$ is the typical 
effective coupling through the intervening strong-disorder phase given by Eq. (2). Here, $x_{i j}$ is the distance separating the spin pairs $i, j$ and $\delta E_{i j}$ is their energy difference. As we iterate this procedure, we generate larger clusters and check whether excitations can resonantly tunnel between any two clusters. Specifically, at any stage in the iterative process, we have a collection of $N$ resonant chains labeled by $j=1, \ldots, N$, each comprised of $\left(m_{j}+1\right)$ bonds. Each resonant cluster has $\approx 2^{m_{j}}$ energy levels, spread over bandwidth $\Lambda_{j}$ equal to the sum of couplings $J$ for the resonant links inside the cluster, and hence, they have typical level spacing $\delta_{a} \approx \Lambda_{j} / 2^{m_{j}}$. The energy levels within a given resonant chain are all strongly admixed and exhibit level repulsion.

If the residual coupling $\Gamma\left(x_{i j}\right)$ between two clusters $i, j$ can resonantly drive transitions among the levels, then we merge these two resonances into a new, larger one. The precise rule for whether or not to merge two resonances depends on the relative size of the bandwidths and level spacings for each. Consider two resonant clusters, labeled $j=1,2$. Without loss of generality, assume that cluster 1 has larger bandwidth $\Lambda_{1}>\Lambda_{2}$. There are two distinct cases to consider. If the level spacing of the first cluster exceeds the bandwidth of the second, $\delta_{1}>\Lambda_{2}$ [Fig. 4], then the minimum energy difference associated with changing the states of both resonances is $\delta E_{1,2}=\delta_{1}-\Lambda_{2}$. On the other hand, if $\delta_{1}<\Lambda_{2}$ [Fig. 4], then the minimum energy cost for transitions is of order $\delta E_{1,2} \approx \delta_{1} \delta_{2} / \Lambda_{2}=\Lambda_{1} / 2^{m_{1}+m_{2}}$.

If $\Gamma\left(x_{12}\right) \ll \delta E_{1,2}$, then the residual coupling between the two resonant clusters merely weakly perturbs the energy levels of each. In this case, the combined energy levels of both clusters are well described by the tensor product of the independent levels associated with each cluster, indicating that energy excitations are well localized

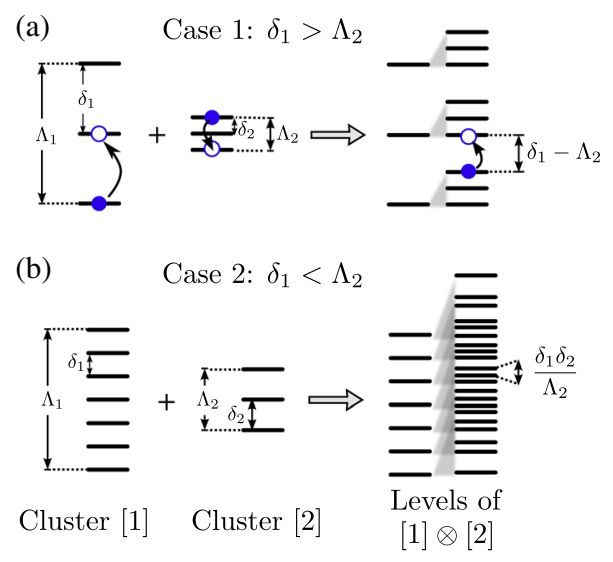

FIG. 4. Two cases for merging resonances. The energy cost for changing the state of one cluster (blue circle to open circle connected by black arrow) can be partially compensated by changing the state of another cluster. The reduced energy penalty, $\delta E_{12}$, for such "flip-flop" transitions is either $\delta_{1}-\Lambda_{2}$ or $\delta_{1} \delta_{2} / \Lambda_{2}$ depending on whether $\delta_{1} \gtrless \Lambda_{2}$, as shown in panels $(a)$ and $(b)$, respectively. independently in each cluster. On the other hand, if $\Gamma\left(x_{12}\right) \gg \delta E_{1,2}$, then the energy levels of both resonances are strongly admixed such that excitations are spread throughout both clusters, and energy can resonantly shuttle between them. In our numerical procedure, we take $\delta E$ as a sharp cutoff, merging resonances coupled by $\Gamma\left(x_{i j}\right)>\delta E_{i j}$.

\section{B. Renormalization of intercluster couplings}

In this appendix, we explain the conceptual necessity of renormalizing the intercluster couplings in the effective resonance percolation model for the MBL transition. Perhaps surprisingly, we find that even though the renormalization steps are conceptually necessary to obtain a well-defined transition in asymptotically large systems, in practice, they may be omitted without changing the universal scaling properties obtained from finite-size scaling in moderately large systems.

If one omits the renormalization of intercluster couplings and simply takes bare couplings, $\Gamma_{i j}=V e^{-x_{i j} / x_{0}}$, where $x_{i j}$ is the distance between clusters $i$ and $j$, there is, in principle, a rare-event-driven breakdown of the strongdisorder phase, which rounds the transition in the effective resonance percolation model [37,38]. Namely, consider the case where one finds a resonant cluster of $N_{*}$ spins, such that the bandwidth of this cluster $\Lambda_{N_{*}}$ is larger than the maximal energy mismatch between two spins $(\approx W)$. Then, with simple geometrically dictated couplings that depend only on the interspin distance, this $N_{0}$ size cluster will deterministically absorb its neighbors and continue to grow in this fashion until it encompasses all spins. Such an "avalanche" style breakdown occurs even for arbitrarily large disorder, as there is always a nonzero probability of finding such an $N_{*}$ spin "seed" cluster.

Specifically, for very strong disorder, $N_{*} \approx(W / V) \gg 1$ spins are required to form a cluster whose bandwidth, $\Gamma_{N_{*}} \lesssim N_{*} V$, exceeds the energy mismatch $W$. The probability of finding such an $N_{*}(W)$ spin resonant cluster is $P_{\text {avalanche }} \approx e^{-W / V}$, indicating that an avalanche will occur for systems larger than $L_{\text {avalanche }} \approx e^{W / V}$. While this system size is extremely large for strong disorder $W \gg V$, it nevertheless indicates that the nonrenormalized couplings lead to the unphysical prediction that the MBL phase will always be unstable to such an avalanche-style breakdown, and it shows that the intercluster renormalization steps are necessary to obtain an asymptotically well-defined phase transition. This avalanche instability is eliminated by proper renormalization of intercluster couplings. Again working in the limit of strong disorder, we observe that, as the "seed" cluster is formed, its couplings to neighboring spins will be typically renormalized downward by a factor of $V / W$ for each spin in the "seed" cluster. Hence, barring an extensive set of accidental resonances, which has vanishing probability for large systems, the avalanche will be suppressed for $(V / W) \ll 1$. 


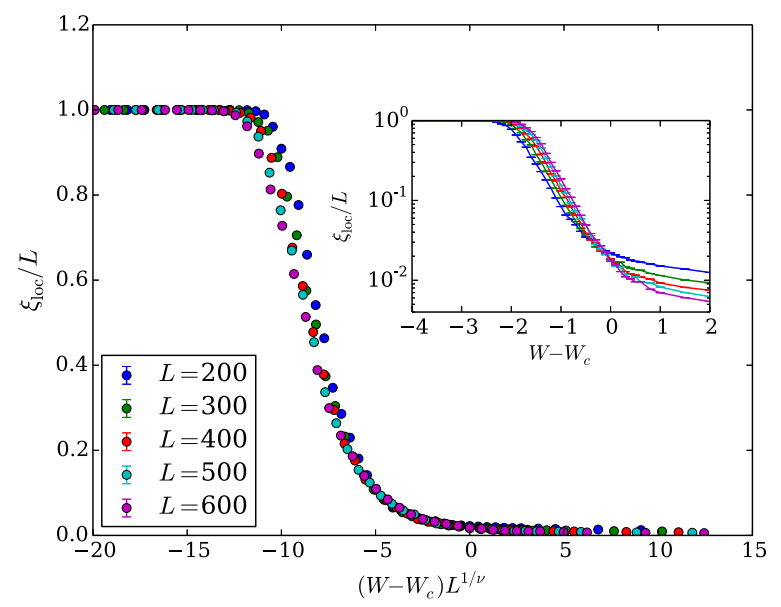

FIG. 5. Finite-size scaling for the disorder-averaged localization length $\xi_{\text {loc }}$ (see Fig. 2) using a simplified model that ignores the renormalization of the couplings. Results are averaged over $\sim 10^{4}$ disorder realizations.

On the other hand, simply omitting the renormalization steps and proceeding with the bare couplings, we obtain essentially identical finite-size scaling results compared to the case with renormalized couplings (Fig. 5). In particular, we obtain finite-size scaling curves for $\xi_{\text {loc }} / L$ that cross nicely at an apparent critical disorder strength $\left(W_{c} / V\right) \approx$ 17 with $V=0.3$. While the precise (nonuniversal) value of $W_{c}$ differs in the absence of renormalization steps, the universal scaling structure such as the crossing value $\left.\left(\xi_{\text {loc }} / L\right)\right|_{W_{c}} \approx 2 \times 10^{-2}$ and correlation-length exponent $\nu \approx 3.5 \pm 0.4$ agree within error bars with those results presented in the main text.

Thus, the theoretically predicted avalanche phenomenon appears to be completely absent even for relatively large $\left(L \approx 10^{3}\right)$ system sizes. In fact, at the MBL transition point $\left(W_{c} / V\right) \approx 17$, from the arguments above, the avalanche instability would only be visible for system sizes of $L \gtrsim e^{17}$ spins, which is completely unaccessible by either numerical simulations or, for that matter, cold atom experiments. Hence, while the renormalized couplings are in principle crucial for obtaining a well-defined transition, in practice one may omit the computationally expensive renormalization step and still observe universal critical finite-size scaling behavior in large systems. This situation is somewhat analogous to accessing critical properties of a conventional quantum critical point at very small, but finite temperature, $T \approx e^{-W_{C} / V}$, which cuts off the flow towards the universal scaling regime, but only above a very large length scale, below which it is possible to accurately identify the universal scaling exponents.

\section{Construction of transport path}

Since we have seen that the potential avalanche instability is absent for computationally accessible system sizes at the critical point $\left(W=W_{c}\right)$, we omit the inconvenient

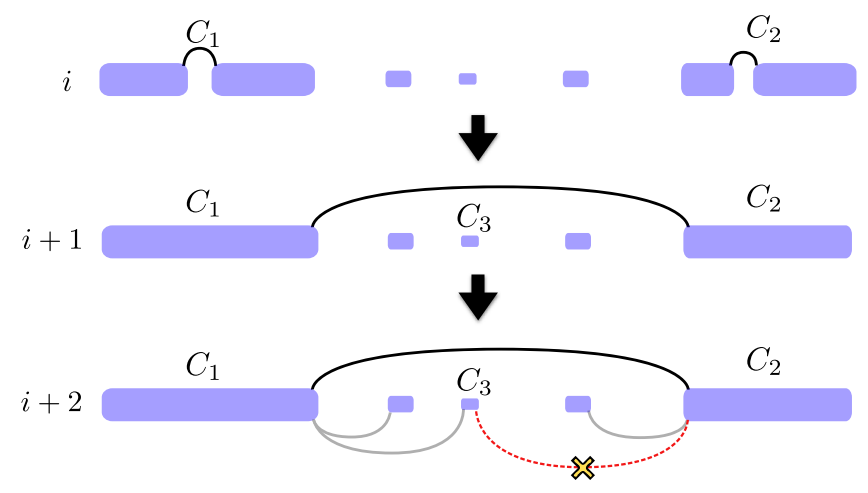

FIG. 6. Transport path through resonant cluster $\left(W<W_{c}\right)$. Schematic depiction of the sequence of resonance-merging steps $(i, i+1, i+2)$ that produces a gap in the transport path through the resonant cluster. See text for a detailed explanation. Blue rectangles are resonantly linked clusters of spins. Black and gray lines indicate resonant links between previously formed clusters. The dashed crossed-out red line indicates a spurious connection that should not be added to the transport path of the cluster.

renormalization steps in computing transport properties. However, in this case, some care is required to properly construct the transport path through the resonant cluster and to avoid a related avalanche-style breakdown of the transport calculation for $W<W_{c}$. Namely, despite the absorption of all bonds into the resonant cluster for $W<W_{c}$, not all bonds contribute equally to transport, and some care is required to properly identify the transport path through the resonant cluster. An illustrative example is shown in Fig. 6.

At some intermediate step, $i-1$, in the resonancemerging process, small resonantly linked clusters of spins (blue rectangles) of various sizes have been identified. At step $i$, smaller clusters merge to form two large resonantly linked clusters, $C_{1}, C_{2}$. In the next step, $i+1$, after accounting for the reduced level spacing of the newly formed clusters, one may find that $C_{1}$ and $C_{2}$ can resonantly exchange energy via the black link, and they are merged into a single larger cluster $C_{1} \cup C_{2}$. It frequently happens that, at step $i+1$, the level spacing of $C_{1}$ (or $C_{2}$ ) alone is too large to facilitate the resonant exchange of energy between smaller clusters, e.g., $C_{3}$, between $C_{1}$ (or $C_{2}$ ), but the combined level spacing of $C_{1} \cup C_{2}$ is small enough to resonate with $C_{3}$. Then, in the subsequent step, $i+2, C_{1} \cup C_{2}$ will absorb the intervening spins. However, it is crucial to note that $C_{3}$ cannot (in this example) resonantly exchange energy with $C_{1}$ or $C_{2}$ alone but rather only by collectively exciting both $C_{1}$ and $C_{2}$. Hence, resonantly transferring energy between $C_{3}$ and $C_{1} \cup C_{2}$ takes at least as long as the time $\tau\left(L_{12}\right)$ to tunnel between $C_{1} \leftrightarrow C_{2}$ (long black link).

If we account for the renormalization of intercluster couplings, this effect is automatically taken into account. However, the renormalization of couplings leads to a large separation of scales in the stochastic matrix $M$ (see Sec. V), which exacerbates numerical precision limits on simulating 
the random walk for long times upon exponentiating $e^{M t}$ (this constraint becomes increasingly detrimental near the critical point because of the critical slowing down of dynamics). To minimize these precision issues, we simply omit the renormalization, bolstered by the observation that the other universal properties are insensitive to this renormalization in the accessible system sizes. However, if we omit the renormalization steps and just blindly connect $C_{3}$ to both $C_{1}$ and $C_{2}$ (e.g., including the crossed-out red line in Fig. 6) in the transport path through the resonant cluster, then we would spuriously allow energy to hop from $C_{1} \rightarrow C_{3} \rightarrow C_{2}$ in time $\tau\left(L_{13}\right)+\tau\left(L_{32}\right) \ll$ $\tau\left(L_{12}\right)$, whereas in this example we have seen that transferring an excitation from $C_{3}$ to $C_{1} \cup C_{2}$ must take at least $\tau\left(L_{12}\right)$. A minimal trick to avoid this unphysical "shorting out" of such insulating gaps in the transport path is to record only a single connection between the closest pair of bonds on $C_{3}$ and $C_{1} \cup C_{2}$ (gray lines in Fig. 6).

\section{APPENDIX B: RENORMALIZATION OF LONG-RANGE INTERACTIONS}

In this appendix, we flesh out the argument alluded to in the main text, regarding the generalization of our results to systems with long-range interactions and critical analogs of MBL systems, dubbed quantum critical glasses (QCGs) in Ref. [15]. Examples of QCGs include phase transitions between MBL phases with different (discrete) symmetry or topological order [13-15,25], as well as stable excited-state critical phases [15]. At strong disorder, QCGs are characterized by a stretched exponential tunneling in which energy $E$ scales with distance $x$ as $E \sim e^{-\left(x / x_{0}\right)^{\psi}}$, with universal exponent $\psi<1$ whose value depends on the universality class of the QCG in question. As for the MBL case, upon weakening disorder, one expects a continuous delocalization transition from QCG to thermal liquid in which resonant clusters percolate.

Whereas the renormalization of intercluster couplings plays a small role in the scaling properties of the MBL transition, we expect it may play a more crucial role in the QCG delocalization transition. Loosely speaking, two clusters with number of spins $n_{1,2}$, respectively, can interresonate if their coupling $\Gamma_{12}$ exceeds the many-body level spacing on the clusters, $\approx 2^{-\left(n_{1}+n_{2}\right)}$. As the clusters are formed, the bare coupling between them, $\Gamma_{0}\left(x_{12}\right) \approx$ $e^{-\left(x_{12} / x_{0}\right)^{\psi}}$ (where $x_{12}$ is the intercluster distance), is perturbatively reduced by a factor of $\approx\left(e^{-\left(x_{\text {typ }} / x_{0}\right)^{\psi}} / \Delta_{\text {typ }}\right)^{n_{1}+n_{2}}$, where $x_{\mathrm{typ}}$ is the typical spacing of spins in the clusters and $\Delta_{\text {typ }}$ is their typical energy mismatch. At the delocalization transition, one expects the clusters to just barely resonate, i.e., $n_{1}+n_{2} \approx x_{12}$, giving effective intercluster coupling $\Gamma_{12} \approx e^{-x_{12} / \tilde{\ell}} \Gamma_{0}\left(x_{12}\right)$, where $\tilde{\ell}^{-1} \approx\left(x_{\text {typ }} / x_{0}\right)^{\psi}-\log \Delta_{\text {typ }}$. As the exponential prefactor dominates over the stretched exponential bare coupling $\Gamma_{0}$, this indicates that the renormalization of couplings gives effective exponential-in-distance interactions between large resonant clusters in QCGs near their delocalization transition, in contrast to their longer-range stretched-exponential-in-distance interactions between individual spins. This suggests that the QCG delocalization transition is actually in the same universality class as the MBL transition. The above scenario contrasts an alternative picture of the QCG-delocalization transition as being driven by chunks of perfect metal interacting through QCG regions via stretched-exponential-in-distance couplings, which would predict an absence of subdiffusion in the delocalized phase [19]. The above considerations instead suggest that the "metallic" clusters in this picture are better thought of as "critical" clusters and that they interact with highly renormalized exponential-in-distance interactions.

We note that similar arguments can be used to suggest the stability of QCGs with stretched exponential interactions, MBL phases with (short-ranged) power-law interactions, $V(x) \sim 1 / x^{p}$, and MBL in higher dimensions for strong disorder. Loosely speaking, though the two-spin interaction $V(L)$ between spins of scale $L$ is much larger than the many-body level spacing $\delta(L) \sim 2^{-L^{d}}$ (where $d$ is the number of spatial dimensions) for large $L$, in order to access the small many-body level spacings, $\sim L^{d}$ powers of the two-spin interactions must be used. For strong disorder, each two-body transition is energy mismatched such that the many-body matrix element $\Gamma(L)$ corresponding to transitions with level spacing $\delta(L)$ also scales as $\Gamma(L) \sim$ $(V / W)^{L^{d}}$, allowing for a MBL phase for $W \gg V$. Similar arguments to those above suggest that the MBL transition with extended interactions will be of the same universality class as that with strictly short-ranged interactions because of the renormalization of the interactions between resonant clusters. We leave a more detailed study of these issues for future work.

[1] L. Fleishman and P. Anderson, Interactions and the Anderson Transition, Phys. Rev. B 21, 2366 (1980).

[2] I. Gornyi, A. Mirlin, and D. Polyakov, Interacting Electrons in Disordered Wires: Anderson Localization and Low- $t$ Transport, Phys. Rev. Lett. 95, 206603 (2005).

[3] D. M. Basko, I. L. Aleiner, and B. L. Altshuler, MetalInsulator Transition in a Weakly Interacting Many-Electron System with Localized Single-Particle States, Ann. Phys. (Amsterdam) 321, 1126 (2006).

[4] A. Pal and D. A. Huse, Many-Body Localization Phase Transition, Phys. Rev. B 82, 174411 (2010).

[5] M. Serbyn, Z. Papić, and D. A. Abanin, Local Conservation Laws and the Structure of the Many-Body Localized States, Phys. Rev. Lett. 111, 127201 (2013).

[6] D. A. Huse, R. Nandkishore, and V. Oganesyan, Phenomenology of Fully Many-Body-Localized Systems, Phys. Rev. B 90, 174202 (2014).

[7] B. Bauer and C. Nayak, Area Laws in a Many-Body Localized State and Its Implications for Topological Order, J. Stat. Mech. 2013, P09005 (2013). 
[8] D. A. Huse, R. Nandkishore, V. Oganesyan, A. Pal, and S. L. Sondhi, Localization-Protected Quantum Order, Phys. Rev. B 88, 014206 (2013).

[9] Y. Bahri, R. Vosk, E. Altman, and A. Vishwanath, Localization and Topology Protected Quantum Coherence at the Edge of 'Hot' Matter, arXiv:1307.4092.

[10] A. Chandran, V. Khemani, C. R. Laumann, and S. L. Sondhi, Many-Body Localization and Symmetry-Protected Topological Order, Phys. Rev. B 89, 144201 (2014).

[11] A. C. Potter and A. Vishwanath, Protection of Topological Order by Symmetry and Many-Body Localization, arXiv:1506.00592.

[12] K. Slagle, B. Zhen, Y.-Z. You, and C. Xu, Many-Body Localization of Symmetry Protected Topological States, arXiv:1505.05147.

[13] R. Vosk and E. Altman, Many-Body Localization in One Dimension as a Dynamical Renormalization Group Fixed Point, Phys. Rev. Lett. 110, 067204 (2013).

[14] D. Pekker, G. Refael, E. Altman, E. Demler, and V. Oganesyan, Hilbert-Glass Transition: New Universality of Temperature-Tuned Many-Body Dynamical Quantum Criticality, Phys. Rev. X 4, 011052 (2014).

[15] R. Vasseur, A. C. Potter, and S. A. Parameswaran, Quantum Criticality of Hot Random Spin Chains, Phys. Rev. Lett. 114, 217201 (2015).

[16] Y. Bar Lev, G. Cohen, and D. R. Reichman, Absence of Diffusion in an Interacting System of Spinless Fermions on a One-Dimensional Disordered Lattice, Phys. Rev. Lett. 114, 100601 (2015).

[17] K. Agarwal, S. Gopalakrishnan, M. Knap, M. Müller, and E. Demler, Anomalous Diffusion and Griffiths Effects Near the Many-Body Localization Transition, Phys. Rev. Lett. 114, 160401 (2015).

[18] D. J. Luitz, N. Laflorencie, and F. Alet, Many-Body Localization Edge in the Random-Field Heisenberg Chain, Phys. Rev. B 91, 081103 (2015).

[19] R. Vosk, D. A. Huse, and E. Altman, preceding article, Theory of the Many-Body Localization Transition in One Dimensional Systems, Phys. Rev. X 5, 031032 (2015).

[20] J. Chayes, L. Chayes, D. Fisher, and T. Spencer, Finite-Size Scaling and Correlation Lengths for Disordered Systems, Phys. Rev. Lett. 57, 2999 (1986).

[21] T. Grover, Certain General Constraints on the Many-Body Localization Transition, arXiv:1405.1471.

[22] M. Schreiber, S. S. Hodgman, P. Bordia, H. P. Lüschen, M. H. Fischer, R. Vosk, E. Altman, U. Schneider, and I. Bloch, Observation of Many-Body Localization of Interacting Fermions in a Quasi-Random Optical Lattice, Science 349, 842(2015).
[23] S. Gopalakrishnan and R. Nandkishore, Mean-Field Theory of Nearly Many-Body Localized Metals, Phys. Rev. B 90, 224203 (2014).

[24] J. A. Kjäll, J. H. Bardarson, and F. Pollmann, Many-Body Localization in a Disordered Quantum Ising Chain, Phys. Rev. Lett. 113, 107204 (2014).

[25] R. Vosk and E. Altman, Dynamical Quantum Phase Transitions in Random Spin Chains, Phys. Rev. Lett. 112, 217204 (2014).

[26] R. Nandkishore and D. A. Huse, Many-Body Localization and Thermalization in Quantum Statistical Mechanics, Annu. Rev. Condens. Matter Phys. 6, 15 (2015).

[27] Jens H. Bardarson, F. Pollmann, and J. E. Moore, Unbounded Growth of Entanglement in Models of Many-Body Localization, Phys. Rev. Lett. 109, 017202 (2012).

[28] M. Serbyn, Z. Papić, and D. A. Abanin, Universal Slow Growth of Entanglement in Interacting Strongly Disordered Systems, Phys. Rev. Lett. 110, 260601 (2013).

[29] P. W. Anderson, D. J. Thouless, E. Abrahams, and D. S. Fisher, New Method for a Scaling Theory of Localization, Phys. Rev. B 22, 3519 (1980).

[30] Properly identifying the transport path in this simplified model requires some care; see Appendix A for details.

[31] J. P. Hulin, J. P. Bouchaud, and A. Georges, Strongly Disordered Chain of Impedances: Theoretical Analysis and Numerical Results, J. Phys. A 23, 1085 (1990).

[32] H. Kim and D. A. Huse, Ballistic Spreading of Entanglement in a Diffusive Nonintegrable System, Phys. Rev. Lett. 111, 127205 (2013).

[33] J. P. Ronzheimer, M. Schreiber, S. Braun, S. S. Hodgman, S. Langer, I. P. McCulloch, F. Heidrich-Meisner, I. Bloch, and U. Schneider, Expansion Dynamics of Interacting Bosons in Homogeneous Lattices in One and Two Dimensions, Phys. Rev. Lett. 110, 205301 (2013).

[34] J. Billy, V. Josse, Z. Zuo, A. Bernard, B. Hambrecht, P. Lugan, D. Clement, L. Sanchez-Palencia, P. Bouyer, and A. Aspect, Direct Observation of Anderson Localization of Matter Waves in a Controlled Disorder, Nature (London) 453, 891 (2008).

[35] We note that the quasiperiodic potential used in Ref. [22] may change the universality class of the transition. However, we expect similar scaling structure to the truly random MBL transition, albeit with potentially different numerical values for universal exponents.

[36] N. Y. Yao, C. R. Laumann, S. Gopalakrishnan, M. Knap, M. Müller, E. A. Demler, and M. D. Lukin, Many-Body Localization in Dipolar Systems, Phys. Rev. Lett. 113, 243002 (2014).

[37] E. Altman and D. Huse (private communication).

[38] A. Chandran and C. R. Laumann (private communication). 